\title{
La problématique de l'insolite et du rêve
}

\author{
Wael Ahmed Abdel Aziz Hassan
}

Tout d'abord, on réfléchit sur la difficulté d'analyser des termes abstraits ou à vrai dire, tous les termes abstraits sont difficiles à comprendre. La notion de l'insolite est extrêmement floue bien que le mot "insolite" renvoie comme l'indique son étymologie à la notion de toute chose inhabituelle. De plus, le mot n'est pas employé très souvent et par conséquent il est difficile de donner une seule conception, notamment si on sait que l'usage de cette notion est divers d'un auteur à l'autre.

En s'interrogeant sur ce terme, on essaierai d'aborder quelques points de vue concernant la signification de ce concept. Or, en travaillant à cette recherche, on va constater deux niveaux qui vont en parallèle: en premier lieu, nous abordons l'insolite comme notion. En second lieu, nous soulignons son caractère comme catégorie dramaturgique.

D'abord, la signification du concept d'insolite ne serait-ce pas une notion transitoire concernant la modernité dont parlait Baudelaire? Dans un de ses articles publiés dans le deuxième recueil de ses oeuvres poetiques intitulé Curiosités esthétiques, il voit - comme l'indiue ce titre-la - la beauté dans l'étrangeté spontanée:

(Le beau est toujours bizarre. Je ne veux pas dire qu'il soit volontairement, froidement bizarre, car dans ce cas il serait un monstre sorti des rails de la vie. Je dis qu'il contient toujours un peu de bizarrerie, de bizarrerie naïve, non voulue, inconsciente, et que c'est cette bizarrerie qui le fait être particulièrement le Beau. $)^{l}$.

Ensuite, ledit concept ne serait-ce pas aussi et de la même façon le merveilleux que proclamait André Breton dans le Manifeste du Surréalisme. Breton semble en accord avec Baudelaire, il trouve la beauté dans le merveilleux "le merveilleux est toujours beau, n'importe quel merveilleux est beau, il n'y a même que le merveilleux qui soit beau." ${ }^{2}$. Il l'analyse ainsi:

(Le merveilleux n'est pas le même à toutes les époques; il participe obscurément d'une sorte de révélation générale dont le détail seul nous parvient: ce sont les ruines romantiques, le mannequin moderne ou tout autre symbole propre à remuer la sensibilité humaine durant un temps. Dans ces cadres qui nous font sourire, pourtant se peint toujours l'irrémédiable inquiétude humaine, et c'est pourquoi je les prends en considération, pourquoi je les juge inséparables de quelques productions géniales, qui en sont plus que les autres douloureusement affectées.) $)^{3}$.

Alors, il nous semble à première vue que l'insolite ne serait pas seulement tout ce qui est beau ou nouveau mais encore tout ce qui contient des 
apparences touchant notre sensibilité et par la suite celles-ci ne devraientelles pas être choquantes, fantastiques, absurdes en vue de produire l'étonnement et la surprise?

Quoi qu'il en soit, cela nous conduit à rapprocher ce concept de l'insolite de la définition de Jarry qui prend l'aspect monstrueux "Il est d'usage d'appeler Monstre l'accord inaccoutumé d'éléments dissonants: le Centaure, la chimère se définissent ainsi pour qui ne comprend. J'appelle monstre toute originale inépuisable beauté"4 . Donc, suffirait-il de remplacer "monstre" par "insolite". A cet égard, n'oublions pas que l'un des dessins publié dans $L^{\prime} Y$ magier ${ }^{5}$ et faisant naître cettte définition figure un centaure sous une apparence très bizarre: un être imaginaire moitié homme et moitié animal. C'est un énorme crapaud à plumes et carapace, les pattes arrière sont plamées, celles d'avant sont griffues avec une tête d'homme qui laisse déborder de sa bouche un enfant à demi dévoré, tandis que à gauche, on remarque un homme agenouillé ayant la position de supplication ${ }^{6}$.

D'autre part, ce dessin semble comme une base pour l'affiche d'Ubu roi ${ }^{7}$ qui représente deux paysans polonais ayant les mêmes traits et les mêmes vêtements prenant la même attitude de supplication devant le monstre qui a pris l'apparence du père $U b u$. Par une simple comparaison entre les deux croquis, il apparaît logiquement qu'Ubu est le monstre ou l'objet insolite dont on parle. Notons ici que Jarry montre dans Ubu Roi sa théorie sur le théâtre contemporain selon laquelle "raconter des choses compréhensibles ne sont qu'alourdirl'esprit et fausser la mémoire, tandis que l'absurde exerce l'esprit et fait travailler la mémoire" ${ }^{\prime 8}$.

Pour cela, cette farce paraît comme une critique de la vraisemblance et du réalisme mais encore telle qu'une guerre contre toute les conceptions théâtrales traditionnelles:

(La construction était volontairement illogique et incohérente; les décors étaient réduits à des accessoires ou remplacés par des pancartes, qui "distancent" l'action; les visages étaient masqués, afin d'abolir tout aspect individuel; l'allure des personnages était celle de marionettes et, à l'occasion, un seul acteur représentait une foule; (...). D'un bout à l'autre, le spectateur voyait, comme à travers une loupe, un monde absurde $)^{9}$.

Ajoutons qu'il indique la voie insolite de son théâtre dans un article publié dans le Mercure de France de septembre 1896 et intitulé "De l'inutilité du théâtre au théâtre" où il suggère un théâtre hors de toute convention réaliste ayant le masque "l'effigie du PERSONNAGE" et la marionnette comme des moyens d'un nouveau langage: simples et accessibles à la fois au public. De plus, il assure la supériorité de la marionnette sur les gestes humains qui utilisent la convention "Aussi Jarry se sent-il attiré lui aussi par les marionnettes qui, d'emblée, donnent à la scène une dimension irréaliste, ou 
préconise-t-il le port du masque qui stylise la figure humaine ${ }^{10}$. D'autre part, il réclame l'abolition du décor en trompe-l'oeil:

(Nous ne reviendrons pas sur la question entendue une fois pour toutes de la stupidité du trompe-l'oeil. Mentionnons que ledit trompe-l'oeil fait illusion à celui qui voit grossièrement, c'est-à-dire ne voit pas, et scandalise qui voit d'une façon intelligente et éligente la nature, lui en présentant la caricature par celui qui ne comprend pas. $)^{11}$.

C'est afin d'ouvrir la voie vers un décor laissant l'imagination du spectateur plus libre "s'insurgeant contre l'esthétique naturaliste, il refuse de donner des repères concrets qui permettaient au spectateur de se rapprocher à la réalité"12. D'ailleurs, il s'intéresse aux éléments scéniques tels que les cris, les silences, les chansons, les écriteaux, les mimes. Il les voit des éléments indispensables pour créer le jeu théâtral "Au jeu illusionniste, il substitue le jeu à découvert. Ce sont les acteurs qui apportent sur scène les éléments de décor dont ils ont momentanément besoin ou qui, par leurs gestes, suppléent à un décor inexistant"13.

A côté de la modernité de Baudelaire, le merveilleux de Breton et le monstre de Jarry, on peut jeter la lumière sur cette citation de Freud "Je me trouve dans l'intéressante position de ne pas savoir si ce que j'ai à dire doit être regardé comme quelque chose de familier depuis longtemps

et évident, ou comme quelques chose d'entèirement nouveau et ahurisssant ${ }^{\prime \prime}$.

Cette phrase pourrait s'appliquer à la conception de l'insolite car le théâtre peut-être à la fois évident et ahurissant. Supposons ici qu'on est, tel qu'un spectateur, dans un spectacle théâtral: une scène vide, un décor, des lumières, on est donc en attente de qui va jouer sur cet espace, c'est bien entendu l'acteur. Que fait-il là? Quand il parle ou agit, son discours et ses mouvements ou plûtot sa représentation pour un tel personnage peut être évident ou étonnant. Alors, prenons en compte la réaction des regardants: si ce discours se présente non articulé, fantasmatique et sans explication, l'insolite se révélera et se déplacera à son tour aux spectateurs. C'est donc à tous les niveaux que paraît, au théâtre, "la déréalisation"15 avec son imagination déreglée et ses situations inattendues qui tendent à déconcerter le public.

D'autre part, cette déréalisation est le fait théâtral même. Celui-ci devient un lieu de plusieurs rencontres interférantes: un plateau avec des lieux dramatiques fictifs, des acteurs vivants représentant d'autres personnages, un texte écrit mais proféré sur scène et contenant des métaphores, des sens ambigues, des images, des gestes. Tout cela a pour but de constituer une représentation imaginaire dont résulte le fantasme. A cet égard, on peut se 
référer à l'affirmation d'André Breton concernant les éléments de la théorie de la représentation "Tout porte à croire qu'il existe un certain point de l'esprit d'où la vie et la mort, le réel et l'imaginaire, le passé et le futur, le communicable et l'incommunicable, le haut et le bas cessent d'être perçus contradictoirement"16.

Alors, pourrait-on parler d'un théâtre onirique qui cherche une surréalité dénonçant la réalité. Dans le rêve, il y a des désirs cachés qu'on souhaiterait et voudrait réaliser. C'est pourquoi, nous sommes à la fois spectateurs et soumis à ces rêves mis en scène à travers ce théâtre onirique qui est "évident" et "ahurissant". Rappelons à cet égard, la définition de Freud du fantasme ou plûtot le rêve éveillé:

(Un fantasme flotte, pour ainsi dire, entre trois temps, les trois moments temporels de notre faculté représentative. Le travail psychique part d'une impression actuelle, d'une occasion offerte par le présent, capable d'éveiller un des grands désirs du sujet; de là, il s'étend au souvenir d'un événement d'autrefois, le plus souvent infantile, dans lequel ce désir était réalisé; il édifie alors une situation en rapport avec l'avenir et qui se présente sous forme de réalisation de ce désir, c'est la le rêve éveillé ou le fantasme, qui porte les traces de son origine: occasion présente et souvenir. Ainsi passé, présent et futur s'échelonnent au long du fil continu du désir) ${ }^{17}$.

En effet, c'est justement comme le rêve, le théâtre de l'insolite nie le réel: les gestes, les mots, les voix, les regards sont toujours démesurés par rapport à la réalité. Donc, dans celui-ci, la parole n'est pas dite mais proférée par les acteurs opposant à celle qui circule dans la société et les objets sur scène dépassent la proportion normale. Tout cela produit la déréalisation, renforce l'action théâtrale et représente une forte attirance envers le spectateur. Nous prendrons par exemple un objet ayant un volume exagéré: les jambes du cadavre dans Amédée ou comment s'en débarrasser de Ionesco.

De cette façon, on pourrait dire que le regard que nous portons sur le rêve va en parallèle avec notre conception sur l'insolite: les deux exigent le rejet du réel vers tout ce qui est déconcertant pour le spectateur. Ce dernier se trouve dans l'impossibilité de croire ça, il le voit étonnant 'pose plus", et l'esprit"se satisfait pleinement de ce qui arrive""18.

$\mathrm{Si}$ on rapproche un spectacle fantasmatique ou onirique avec le thêâtre traditionnel, on trouve certaines conventions distinguant le dernier: règle des trois unités (une action ne dépassant pas les vingt-quatre heures - un lieu unique - une intrigue unique), règle de bienséance et de vraisemblance, discours explicite, structure logique, héros traditionnel, décor naturaliste. Un théâtre auquel le spectateur s'habitue et à travers lequel, il se compare toujours avec le héros traditionnel romantique ou tragique et s'identifie avec le personnage interprétant sur scène, il n'est plus donc surpris. Par contre, 
dans le théâtre onirique, le spectateur est toujours étonné. Ici, deux questions s'imposent: pourquoi un tel spectateur devrait, au moins au début de la représentation, rejeter cela comme dérangeant? Et c'est au fil du temps qu'il voit ça admissible? On cite ici un avis de Simone Benmussa ${ }^{19}$ éclairant sur ce point:

(En effet,j'ai dit plus haut que "tout d'abord" le théâtre déconcertait car il attaque les défenses des spectateurs mais, très vite, ceux-ci trouvent des parades, reconstituent leurs défenses et ils peuvent le faire pour deux raisons: La première c'est que tout théâtre déconcertant doit, pour se faire admettre, passer par un certain esthétisme (...) sous peine d'être rejeté totalement, la seconde c'est que les spectateurs, justement, se savent au spectacle $)^{20}$.

Quoi qu'il en soit, si on acceptait un spectacle comme ça ou non, nous serions devant un théâtre d'images qui ne peut s'installer dans aucune structure et s'oppose au discours logique auquel nous nous sommes habitués. Alors, l'insolite ou l'onirisme présente un grand changement par rapport au théâtre traditionnel en mettant le spectateur en grand désordre. C'est du fait qu'il contient en tant qu'un jeu dramatique "une excellente forme de déréalisation: celle où la technique est poussée à un point si extraordinaire de convention que l'absolu de la convention devient pour l'imagination libertéabsolue" 21 .

Cette liberté absolue de l'imagination résulte de l'étrangeté, soit celle du rêve ou à vrai dire le fait théâtral, soit de la mise en scène: ça veut dire la cohésion du jeu de l'acteur, des gestes ${ }^{22}$, de la musique, de la diction et de tous les autres moyens scéniques aboutissant à une déréalisation absolue. Dans le théâtre onirique, tout est déréalisé au service des fantasmes qui sont utilisés pour critiquer les faits de la société. A cet égard, prenons pour exemple Rhinocéros d'Eugène Ionesco qui, à travers la métamorphose des hommes en animaux, représente l'image d'un régime politique autoritaire et totalitaire dans lequel les individus sont soumis et enfermés et pas de place pour la pensée individuelle étant tout à fait combattue et supprimée. Dans cette pièce, Bérenger serait peut-être Ionesco lui-même qui a fui de ce genre de régime et l'a combattu de toutes ses forces pour créer une société civilisée et libre.

De plus, le spectateur serait à travers l'onirisme ou plutôt l'insolite dans le coup: il est au courant et participe à l'action. Mais dans quelle mesure le spectateur fait partie prenante de la représentation? D'abord, on peut dire que lorsqu'il s'agit de théâtre, le spectateur est un élément essentiel de la représentation, sans public pas de théâtre. Mais, si celui-ci est un lieu de plaisir où l'on vient rire avec une pièce comique ou pleurer pendant une tragédie, ce seront seulement les émotions essentielles du rire et des pleurs 
qui permettent d'impliquer le public et de le faire participer au spectacle. Avec ces réactions précises, le message sur scène devient direct, il n'y a qu'un seul sens et le spectateur le reçoit ou ne le reçoit pas, le comprend ou ne le comprend pas, ça ne fait rien. Ainsi, sa participation dans la représentation a une telle passivité.

Cependant, devant un spectacle insolite, le spectateur ne sait plus comment il doit réagir: faut-il rire ou pleurer. Il s'étonne et toujours en désordre, il peut être dérangé, choqué par l'ambiance ambiguë et à la suite de l'étrangeté à laquelle il ne s'habitue pas. L'imagination ou plutôt la déréalisation le fait réfléchir sur ce qui se déroule sur scène et, il devient devant deux choix opposés: celui de l'implication ou celui du refus. Avec cette réaction qui pourrait dépasser la durée du spectacle on peut souligner que la participation ici devient une telle intégration évidente à la représentation qui rend à la pièce son efficacité et sa vivacité. N'oublions pas que l'intrigue onirique de l'auteur dramatique et les procédés artistiques du metteur en scène contribuent à séduire le public et lui faire admettre un spectacle fantasmatique.

De ci-dessus et si le concept de l'insolite renvoie à la déréalisation ou se rapporte, comme l'indique son étymologie, à la notion de l'inhabituel, une question se pose à nous: quand on revoit une pièce de théâtre onirique pour la dixième fois, on peut y trouver encore des choses étonnantes, peut-on garder la même réaction de la première de la pièce? C'est bien entendu non, parce que la sensation de la surprise n'arrive que de la première fois et par la suite on s'y habitue. Ça veut dire qu'il n'est pas possible que le spectateur ait la même perception dix fois de suite quand on connaît à l'avance ce qui va se passer et c'est également la même chose qu'on peut observer chez le lecteur. Supposons que celui-ci se donne à lire un livre qu'il a d'insolite, à la première lecture n'y a -t-il pas un choc extraordinaire commençant à disparaître dès qu'on relit plus d'une fois parce qu'on sait d'avance ce qu'il y a dedans. D'ici, la sensation devient moins vif et assez faible devant le déjà connu. Alors, on peut dire que l'insolite à ce point est limité à la perception au premier abord "La notion d'insolite appartient au domaine de la perception"23.

Soyons plus convaincants: un tel événement, un fait ou une parole ne peut apparaître comme inhabituel ou insolite, comme rompant une habitude que s'il a une relation avec l'habitude qu'il rompt. Ça veut dire qu'un objet isolé n'est pas en soi insolite, c'est sa relation avec d'autres objets qui peut être perçue comme inhabituel. L'insolite est d'abord la perception d'une relation.

Prenons pour exemple, la première oeuvre d'Eugène Ionesco La cantatrice chauve dont la première représentation a eu lieu le 11 mai 1950 au théâtre des Noctambules dans une mise en scène de Nicolas Bataille. Le titre même de cette anti-pièce nous laisse dans l'attente de voir une forme achevée d'un spectacle scénique mais pas d'intrigue, pas d'action, même la présence d'une 
chanteuse ayant une calvitie. Alors, l'insolite ici est perçue à travers deux relations existantes au titre même: la première, c'est la relation entre un spectacle chantant et un autre qui n'est qu'une soirée bourgeoise. La deuxième est celle qui est entre une chanteuse ayant une telle qualité qui est ici la calvitie et notre conception connue de la chanteuse d'opéra ou plutôt la réalité de son apparence comme une femme chanteuse. Un autre exemple, nous le voyons avec la métamorphose de tout un village en rhinocéros: la relation perçue entre un être humain et sa métamorphose en animal, c'est l'insolite.

De cette façon, pourrait-on dire que la définition de l'insolite est la perception d'une manière première et imprévisible de la relation établie entre un événement habituel et ce qui le montre déconertant, étrange, bizarre, alogique ou peut-être ce qu'on a déjà évoqué lorsqu'on parlait de merveillaux ou du fantasme de la déréalisation. Proprement dit, la perception des relations inhabituelles semble comme un fait de rupture. Mais que signifie le mot "rupture"? C'est le fait qu'une action, l'action réelle, est interrompue brusquement sous l'effet d'une autre nouvelle, l'événement imaginaire, qui sort de l'ordinaire et des idées traditionnelles en vue de surprendre et mettre en désordre.

En effet, l'insolite est premièrement une sorte de jugement ou plutôt une réaction qui dépend de la pensée de l'individu. Deuxièmement, il modifie ${ }^{24}$ la représentation de l'événement de l'état réel à l'état inhabituel, donc le même fait, lorsqu'il est répété, ne peut pas avoir deux fois de suite la même qualité de l'étrangeté. C'est parce que la relation perçue entre les deux états de l'action est petit à petit intégrée dans l'esprit de l'individu pour être habituel ou à vrai dire connue d'avant perdant son caractère tel qu'un élément nouveau qui le laisse dans une attente.

On arrive ainsi à mettre en relief un tel point: l'insolite apparaît comme un de moyens qui contribuent à activer et renforcer l'action. Suggérons que nous assistons à une pièce de théâtre dont l'intrigue est présentée d'une manière logique et ordinaire, dans ce cas-là, on reste libre de porter son jugement parce que la logique ne fait pas travailler la pensée, ni attire l'attention. Si au contraire, un tel spectacle a pour principale fonction d'éloigner la compréhension, de masquer la réalité d'une vie moderne stressante à travers l'étrangeté qui déconcerte mais attire et fascine à la fois présentant une manière nouvelle et originale de voir, il oblige notre jugement à être dans une attente d'un réel nouveau, à paraître suspendu entre le refus et l'acceptation. En outre, il nous pousse à en parler plus tard. Pourrait-on dire que l'insolite n'est pas seulement un moyen d'expression mais également un facteur d'activation.

Dans la mesure où on parle d'un théâtre onirique dont la représentation devient plus vive et plus forte par l'insolite et si celui-ci porte sur la 
perception mentale de l'individu, on peut affirmer que dans notre vie quotidienne, nous pouvons nous trouver en face des événements inhabituels perçus comme rupture du normal et qui naissent du hasard ou selon la nécessité du monde réel tels que les catastrophes naturelles: tremblement de terre, inondations, éruptions volcaniques mais encore les expériences de la vie réelle: actions remarquables et exptionnelles ou à vrai dire les exploits. C'est l'insolite qu'on appellerait l'insolite naturel qui n'a pas une signification particulière.

Néanmoins, l'insolite dont il est question dans cette étude et qui se rapporte au jeu de la scène paraît comme provoqué parce qu'il découle essentiellement d'une intrigue écrite par l'auteur, jouée par des acteurs et perçue par les spectateurs. Ce type d'insolite devrait faire preuve d'une telle signification indicative ou plutôt symbolique, chargé d'indications qui renvoient à des éléments de fiction représentés avec des ruptures inhabituelles. D'autre part, il joue le rôle d'une forme de provocation. En nous référant à l'histoire des Mamelles de Tirésias de Guilaume Apollinaire dans laquelle la fantasie de la métamorphose transsexuelle où l'inversion des fonctions naturelles arrive au maximum quand l'auteur propose que le mari métamorphosé en femme accouche dans un seul jour un trop grand nombre d'enfants, nous trouvons que ces actions fantasmatiques présentées avec des ruptures insolites sont chargées d'indications renvoyant à l'intention du poète qui a pour but d'insister sur le poids d'un problème social tel que la baisse de la natalité française.

Prenons un autre exemple: le fameux cadavre grandissant d'Amédée ou comment s'en débarasser d'Eugène Ionesco est un élément insolite parce qu'il cherche à briser une habitude pour introduire un tel facteur d'attente provoquant une rupture qui oppose notre idée du cadavre normal à un autre de ce volume. Si on fait référence au monde réel, nous voyons que les corps des personnes mortes n'ont pas l'habitude de grandir, alors le cadavre comme objet réel ne fabrique pas l'insolite mais son volume démesuré ou déréalisé. Ça veut dire qu'il faut donner une fausse apparence à l'objet pour créer l'insolite. Celui-ci est à la fois significatif "Aux dires mêmes de Ionesco, le cadavre matérialise le péché, la faute originale, le temps qui s'allonge et qui, créant une usure, les sépare de plus en plus." ${ }^{25}$. Ajoutons qu'il y a des quantités de champignons qui ont poussé dans la salle à manger et surtout autour des jambes du mort pour figurer la montée de l'angoisse entre le couple Amédée - Madeleine "Le pourrissement de la salle à manger infestés de champignons, la croissance du cadavre reflètent l'érosion de ses relations avec Madeleine, la mort de leur amour"26.

D'ailleurs, l'insolite indicatif apparaît bien avec l'image d'un homme devenant une bête effrayante et aggressive dans Rhinocéros. Cette pièce porte à la scène l'atrocité de l'occupation allemande en France à travers les 
habitants d'une petite ville de province qui se sont rhinocérosés l'un après l'autre. Rappelons à cet égard la parole de Marie-Claude Hubert en se demandant pourquoi Ionesco choisit le rhinocéros qui vit en solitaire au lieu d'un animal ayant l'instinct d'être en groupe "Tellement hanté par les images de la solitude, Ionesco a choisi un animal solitaire comme symbole des phénomènes d'hystéries collectives" 27.

Cependant, cet insolite chargé d'une intention significatifve se diffère de celui qui veut signifier l'insolite et ne présente aucune autre idée: "C'est l'inhabituel qui déconcerte mais ne renvoie pas à une autre chose, comme par exemple une certaine forme d'absurdité" 28 . A propos de ce point, on peut se référer à la Cantatrice chauve de Ionesco où il veut libérer la scène de toutes les conventions de la réalité en employant un langage vidé de signification impliquant un type de plaisanterie. Cela est bien indiqué dans un dialogue incohérent: $M$. et Mme Martin se rencontrent dans le salon des Smith chez qui ils ont été invités. Ils commencent la conversation en attendant l'arrivée de leurs hôtes comme deux étrangers qui ne se connaissent pas. Parfois, ils se regardent attentivement et avec un certain étonnement " $M$. MARTIN: Mes excuses, madame, mais il me semble, si je ne me trompe, que je vous ai déjà rencontrée quelque part.MME MARTIN: A moi, aussi, Monsieur, il me semble aue je vous ai déjà rencontré quelque part" ${ }^{\prime 2}$.

C'est également évident avec l'emploi de néologismes, termes n'ayant pas de sens que pour l'auteur qui les invente tels que: "kakatoes", "cacade", "cocardard", "caïman". Alors si "La subversion de la raison peut être partielle ou absolue. Le non-sens peut admettre des normes rationnelles, simplement en les bouleversant." ${ }^{\prime 30}$ nous sommes ainsi devant un trouble ou plutôt une absurdité du langage ayant pour but de briser plus encore la communication avec le spectateur qui se met en déconcertation à travers cette absence de logique.

Bien que la Cantatrice chauve apparaisse comme un manifeste où sont contenus les grands principes de la dramaturgie ionescienne et son absurdité ne renvoie pas à une autre signification, certains critiques ont un autre point de vue vis-à-vis ce type de l'insolite:

(Mettant en scène un homme et une femme que la vie commune rend si étrangers l'un à l'autre, Ionesco médite sur ce narcissime indépassable qui enferme l'être dans sa solitude. Vision pessimiste du couple, réflexion amère sur la vanité des relations humaines qui se retrouvera dans toutes les oeuvres ultérieures! $)^{31}$.

\section{Oeuvres dramatiques:}

\section{Références}

- Théâtre I, Gallimard, 1954 (La Cantatrice chauve ; La leçon ; Jacques, ou La soumission; Les chaises ; Victimes du devoir ; Amédée, ou 
Comment s'en débarasser).

II. Journaux intimes: Notes et contre notes, Gallimard, Paris, 1983.

III. Etudes critiques:

- ABIRACHED Robert, La crise du personnage dans le théâtre moderne, Gallimard, 1994.

- BAUDELAIRE Charles, Oeuvres poétiques, volume II "Curiosités esthétiques", Michel Lévy Frères, 1868, partie IV intitulé: Exposition universelle, 1855, Beaux Arts, article $\mathrm{n}^{\mathrm{O}} \mathrm{I}$ intitulé "Méthode de critique - de l'idée moderne du progrès appliquée aux Beaux-Arts - déplacement de la vitalité.".

- BRETON André, Le manifeste du surréalisme, Gallimard, 1924.

- Colloques de Strasbourg, L'onirisme et l'insolite dans le théâtre contemporain, textes recueillis par Paul Vernois, Klincksieck, 1974.

- EVRARD Franck, le théâtre français du XX ${ }^{\mathrm{è}}$ siècle, ellipses, 1995.

- Freud Sigmund, La création littéraire et le rêve éveillé, un document produit en version numérique Par Jean-Marry Tremplay, l'article originalement publié en 1908.

- HUBERT Marie-Claude, Eugène Ionesco, Editions du Seuil, 1990.

- HUBERT Marie-Claude, Les grandes théories du théâtre, Armand Colin, 1998.

- LAUBREAUX Raymond, Les critiques de notre temps et Ionesco, Editions Garnier Frères, 1973.

- Surer Paul, Cinquante ans de théâtre, société d'édition d'enseignement supérieur, Paris, 1969, p. 330.

${ }^{11}$ Baudelaire Charles, Oeuvres poétiques, volume II "Curiosités esthétiques", Michel Lévy Frères, 1868, partie IV intitulé: Exposition universelle, 1855, Beaux Arts, article no I intitulé "Méthode de critique - de l'idée moderne du progrès appliquée aux Beaux-Arts déplacement de la vitalité.", pp. 211-244. Cf. P. 216.

${ }^{2}$ Breton André, Le manifeste du surréalisme, Gallimard, Paris, 1924, p.7.

${ }^{33}$ Ibid.

${ }^{4}$ Béhar Henri, La dramaturgie d'Alfred Jarry, dans colloque de Strasbourg, textes recueillis et présentés par Paul Vernois intitulés "L'onirisme et l'insolite dans le théâtre français contemporain", Paris, éd. Klincksieck, 1974, p. 5.

${ }^{5}$ En octobre 1894, Le Mercure de France a annoncé la naissance d'une revue rédigée par Remy Gourrmont et Alfred Jarry qui ne participe que pour les cinq numéros d'entre les huit d'octobre 1894 à décembre 1896. Le but de cette revue est de porter un regard neuf sur l'histoire de l'art en privilégiant l'imagerie ancienne, folklorique et légendaire. Mais pourquoi un y à ce mot, quand les dictionnaires disent imagier?», écrivaient-ils. Réponse: parce qu'«aujourd'hui comme jadis», on peut réclamer

«un peu de liberté dans l'orthographe, pour un peu plus de beauté».

${ }^{6} \mathrm{Cf}$. l'illustration de la fin de la recherche.

${ }^{7}$ Une énorme farce épique jouée en 1896. La pièce se déroule dans un concert de cris d'oiseaux et de siffles, elle provoque les protestations de la critique traditionnelle. C'est la 
première et la plus spectaculaire manifestation du théâtre d'avant-garde. C.f. Evrard Franck, Le théâtre français du XXè siècle, ellipses, 1995, Programme d'Ubu roi, p. 16.

${ }^{8}$ Surer Paul, Cinquante ans de théâtre, société d'édition d'enseignement supérieur, Paris, 1969 , p. 330.

${ }^{9}$ Ibid.

${ }^{10}$ Hubert Marie-Claude, Les grandes théories du théâtre, Armand Colin, Paris, 1998, p. 216.

${ }^{11} \mathrm{Ibid}$. Cf. l'article de Jarry intitulé De l'inutilité du théâtre au théâtre, p. 215.

${ }^{12}$ Evrard Franck, Le théâtre français du XXè siècle, ellipses, 1995, p. 14.

${ }^{13}$ Hubert Marie-Claude, Les grandes théories du théâtre, Op. Cit., p. 217.

${ }^{14}$ Benmussa Simone, La déréalisation par la mise en scène, dans colloque de Strasbourg, textes recueillis et présentés par Paul Vernois, Op. Cit., p. 27.

${ }^{15}$ Ibid. P. 28.

${ }^{16}$ Abirached Robert, la crise du personnage dans le théâtre moderne, Gallimard, 1994. P. 317,318 .

${ }^{17}$ Freud Sigmund, La création littéraire et le rêve éveillé, un document produit en version numérique Par Jean-Marry Tremplay, p. 7. L'article originalement publié en 1908.

${ }^{18}$ Abirached Robert, La crise du personnage dans le théâtre moderne, Op. Cit., p. 318.

${ }^{19}$ Metteur en scène, dramaturge et écrivaine française, née à Tunis en 1931 et morte à Paris en 2001

${ }^{20}$ Benmussa Simone, La déréalisation par la mise en scène, dans colloque de Strasbourg, textes recueillis et présentés par Paul Vernois, Op. Cit., P. 34.

${ }^{21}$ Etiemble René, discussion sur la communication de Mme Simone Benmussa dans colloque de Strasbourg, textes recueillis et présentés par Paul Vernois, Op. Cit. P. 39.

${ }^{22}$ Rappelons ici les propos de Ionesco publiés dans son livre Notes et contre notes : "Tout est langage au théâtre, les mots, les gestes, les objets, l'action elle-même car tout sert à exprimer, "Cf. Ionesco Eugène, Notes et contre notes, Gallimard, Paris, 1983, p. 116.

${ }^{23}$ Voltz Pierre, L'insolite est-il une catégorie dramaturgique?, dans L'onirisme et l'insolite dans le théâtre français contemporain, textes reueillis et présentés par Paul Vernois, $O p$. Cit., P. 49.

${ }^{24}$ On ne dit pas "change" parce qu'on parle ici d'une transformation sans changement complet.

${ }^{25}$ Hubert Marie-Claude, Eugène Ionesco, Editions du Seuil, 1990, p.118.

${ }^{26}$ Ibid., p. 124.

${ }^{27}$ Hubert Marie-Claude, Eugène Ionesco, Op.Cit, p. 141.

${ }^{28}$ Voltz Pierre, L'insolite est-il une catégorie dramaturgique, colloque de Strasbourg, Op. Cit. p. 54.

${ }^{29}$ Ionesco Eugène, Théâtre I: La Cantatrice Chauve; La leçon, Jacques ou la soumission; Les chaises; Victimes du devoir; Amédée ou comment s'en débarasser, Gallimard, 1954, p. 26.

${ }^{30}$ I. Grossvogel David, Ionesco et l'Absurde, dans Les critiques de notre temps et Ionesco, présentation par Raymond Laubreaux, Editions Garnier Frères, 1973, p. 26.

${ }^{31}$ Hubert Marie-Claude, Eugène Ionesco, Op.Cit, p. 60. 